\title{
Development of autoantibodies against muscle- specific FHL1 in severe inflammatory myopathies
}

\author{
Inka Albrecht, ${ }^{1}$ Cecilia Wick, ${ }^{1}$ Åsa Hallgren, ${ }^{2}$ Anna Tjärnlund, ${ }^{1}$ Kanneboyina Nagaraju, ${ }^{3}$ Felipe Andrade, ${ }^{4}$ Kathryn Thompson, ${ }^{3}$ \\ William Coley, ${ }^{3}$ Aditi Phadke, ${ }^{3}$ Lina-Marcela Diaz-Gallo,, Matteo Bottai, ${ }^{1}$ Inger Nennesmo, ${ }^{6}$ Karine Chemin, ${ }^{1}$ Jessica Herrath, ${ }^{1}$ \\ Karin Johansson, ${ }^{1}$ Anders Wikberg, ${ }^{1}$ A. Jimmy Ytterberg, ${ }^{1,7}$ Roman A. Zubarev, ${ }^{7}$ Olof Danielsson, ${ }^{8}$ Olga Krystufkova, ${ }^{9}$ \\ Jiri Vencovsky, ${ }^{9}$ Nils Landegren, ${ }^{2,10}$ Marie Wahren-Herlenius, ${ }^{11}$ Leonid Padyukov, ${ }^{1}$ Olle Kämpe, ${ }^{2,10}$ and Ingrid E. Lundberg ${ }^{1}$ \\ ${ }^{1}$ Rheumatology Unit, and ${ }^{2}$ Experimental Endocrinology, Department of Medicine (Solna), Karolinska Institutet, Stockholm, Sweden. ${ }^{3}$ Children's National Medical Center, \\ Center for Cenetic Medicine Research (CGMR), Washington, DC, USA. ${ }^{4}$ Department of Medicine, Johns Hopkins University School of Medicine, Baltimore, Maryland, USA \\ ${ }^{5}$ Unit of Biostatistics, Institute of Environmental Medicine, ${ }^{6}$ Department of Laboratory Medicine, and ${ }^{7}$ Department of Medical Biochemistry and Biophysics, Karolinska Institutet, Stockholm, Sweden. \\ ${ }^{8}$ Department of Clinical and Experimental Medicine, Division of Neurology, Faculty of Health Sciences, Linköping University, Linköping, Sweden. ${ }^{9}$ Institute of Rheumatology and \\ Department of Rheumatology, First Faculty of Medicine, Charles University, Prague, Czech Republic. ${ }^{10}$ Science for Life Laboratory, Department of Medical Sciences, Uppsala University, Uppsala, Sweden. \\ ${ }^{11}$ Experimental Rheumatology Unit, Department of Medicine (Solna), Karolinska Institutet, Stockholm, Sweden.
}

\begin{abstract}
Mutations of the gene encoding four-and-a-half LIM domain 1 (FHL1) are the causative factor of several X-linked hereditary myopathies that are collectively termed FHL1-related myopathies. These disorders are characterized by severe muscle dysfunction and damage. Here, we have shown that patients with idiopathic inflammatory myopathies (IIMs) develop autoimmunity to FHL1, which is a muscle-specific protein. Anti-FHL1 autoantibodies were detected in $25 \%$ of IIM patients, while patients with other autoimmune diseases or muscular dystrophies were largely anti-FHL1 negative. Anti-FHL1 reactivity was predictive for muscle atrophy, dysphagia, pronounced muscle fiber damage, and vasculitis. FHL1 showed an altered expression pattern, with focal accumulation in the muscle fibers of autoantibody-positive patients compared with a homogeneous expression in anti-FHL1-negative patients and healthy controls. We determined that FHL1 is a target of the cytotoxic protease granzyme B, indicating that the generation of FHL1 fragments may initiate FHL1 autoimmunity. Moreover, immunization of myositis-prone mice with FHL1 aggravated muscle weakness and increased mortality, suggesting a direct link between anti-FHL1 responses and muscle damage. Together, our findings provide evidence that FHL1 may be involved in the pathogenesis not only of genetic FHL1-related myopathies but also of autoimmune IIM. Importantly, these results indicate that anti-FHL1 autoantibodies in peripheral blood have promising potential as a biomarker to identify a subset of severe IIM.
\end{abstract}

\section{Introduction}

Idiopathic inflammatory myopathies (IIMs) are a heterogeneous group of rare systemic autoimmune diseases collectively called myositis, which causes progressive muscle weakness. Several forms of the disease, including polymyositis (PM), dermatomyositis (DM), inclusion body myositis (IBM), and immune-mediated necrotizing myopathy can be distinguished on the basis of clinical features, muscle histopathology, and autoantibody profiles (1-4). For IBM, muscle-specific autoantibodies against cytosolic 5'-nucleotidase $1 \mathrm{~A}$ (cN-1A) (5-7) and desmin (8) were recently described as serological biomarkers for this disease subtype. Interestingly, myositis-specific autoantibodies described in PM and DM are directed against ubiquitously expressed intracellular proteins (9-11) and show a lack of muscle specificity. Identification of novel muscle-specific targets involved in immune-mediated processes and their detailed char-

Conflict of interest: Inka Albrecht, Cecilia Wick, Åsa Hallgren, Olle Kämpe, and Ingrid E. Lundberg have filed a patent application related to this work on a diagnostic test to identify patients with anti-FHL1 autoantibodies.

Submitted: January 20, 2015; Accepted: September 25, 2015.

Reference information: / Clin Invest. 2015;125(12):4612-4624. doi:10.1172/JCI81031. acterization will facilitate the understanding of the initiation and perpetuation of chronic autoimmune attacks on the skeletal muscle.

FHL proteins are characterized by four-and-a-half highly conserved LIM domains, which mediate protein-protein interactions. FHL1 is predominantly expressed in the skeletal muscle, and, although its precise function is not known, there is experimental evidence showing that FHL1 is involved in muscle growth (12), differentiation $(13,14)$, and structural maintenance such as sarcomere assembly (15). FHL1 is further described to be involved in cell signaling pathways including Smad/TGF- $\beta$-like- (16), estrogen- (17), Notch- (18), and MAPK (19) cascades. Several spliced variants of FHL1 have been identified as containing additional domains with different localization patterns and tentatively coding for protein variants with different functions (20). Importantly, genetic FHL1 mutations are causative for various rare X-linked myopathies that mostly appear in youth; these include reducing body myopathy (RBM) (21-24), X-linked myopathy characterized by postural muscle atrophy (XMPMA) $(25,26)$, scapuloperoneal myopathy (SPM) (27), and Emery-Dreifuss muscular dystrophy (EDMD) (28). These FHL1-associated myopathies share pathological features, i.e., severe muscular dysfunction and damage, but 
A

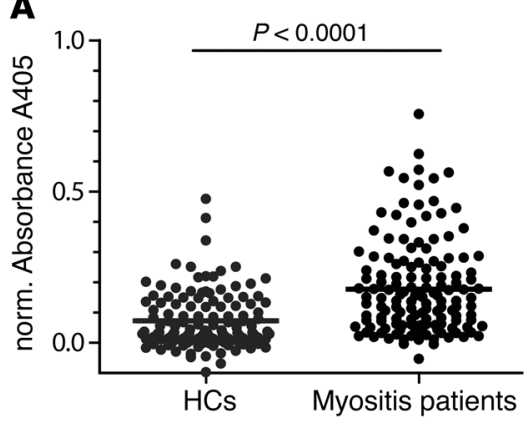

B

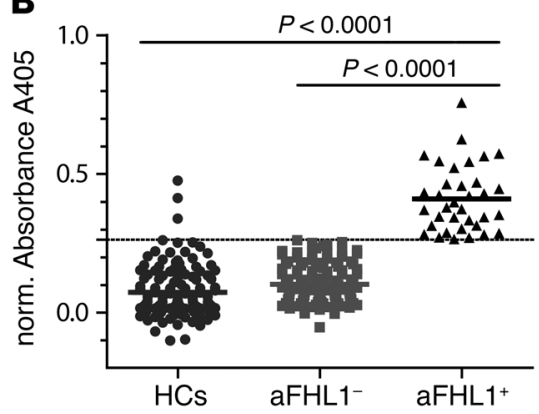

C

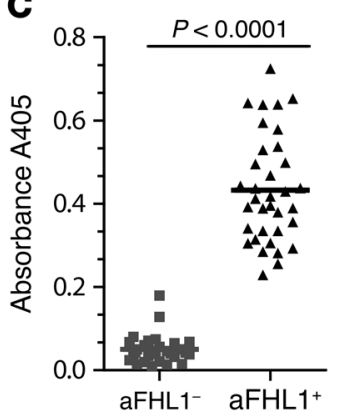

D

$\mathrm{aFHL} 1^{+}$patient sera no.

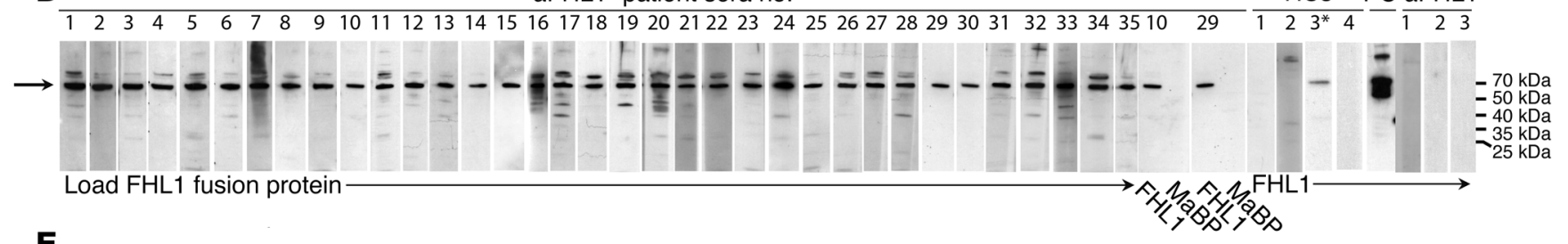

E

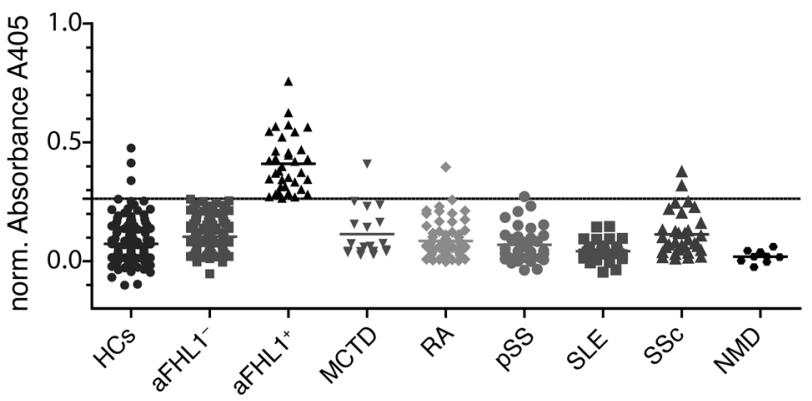

Figure 1. IIM patients have anti-FHL1 autoantibodies that are specific to this disease. (A) Sera from patients with IIM (PM, DM, or IBM; $n=141)$ were analyzed by ELISA for reactivity to recombinant FHL1-MaBP fusion protein and compared with sera from sex- and age-matched HCs ( $n=126)$. (B) A cutoff value was calculated, allowing subdivision of the patients into anti-FHL1- (aFHL1-) and anti-FHL1+ patients (cutoff $=$ mean [norm. absorbance $\mathrm{HC}$ ] $+2 \times$ $\mathrm{SD}=0.26228$ ). (C) Anti-FHL1 positivity was confirmed by another ELISA using recombinant His-tagged FHL1 and by comparing anti-FHL1 ${ }^{+}(n=35)$ with sexand age-matched anti-FHL1- patients $(n=30)$ as well as by Western blotting $(\mathbf{D})$ using recombinant FHL1-MaBP fusion protein. All 35 of the anti-FHL1+ patients were analyzed (lanes 1-35). Lanes show reactivity of sera from anti-FHL1+ patients 10 and 29 to MaBP loaded next to FHL1-MaBP, of sera from 4 $\mathrm{HCs}$ (sera $3^{*}$ with positive reactivity detected by ELISA in B), of sera from a positive control (PC) using a commercial anti-FHL1 antibody, and of sera from 3 anti-FHL1- patients. (E) Reactivity to FHL1 in sera from HCs and IIM patients was compared by ELISA with anti-FHL1 reactivity in sera from patients with MCTD $(n=19)$, RA $(n=67)$, pSS $(n=35)$, SLE $(n=33)$, and SSc $(n=32)$, as well as in sera from patients with neuromuscular disease (NMD; $n=9)$. Statistical analysis for A-C was performed using a 2-tailed Mann Whitney $U$ test; each data point represents 1 individual, and horizontal bars indicate the mean values. For A, B, and E, normalized A405 values (norm. A405 $=$ FHL1-MaBP-A $A_{405}$ minus MaBP-A ${ }_{405}$ ) are shown.

may differ in their extent of muscle weakness and site of major symptoms (29). The most severe forms of FHL1-associated myopathies result in complete loss of ambulation and death caused by respiratory or heart failure (29). A detailed molecular link between FHL1 mutations and these muscular symptoms has not been elucidated, but it has been suggested to include protein instability, consequently leading to protein dysfunction, aggregation, and degradation (23). Together, these studies indicate that FHL1 is critical for normal skeletal muscle structure and function.

In the current study, we aimed to screen for a muscle-specific autoantigen using a muscle-specific cDNA library. We found autoantibody reactivity to FHL1 with high specificity for IIM and demonstrated a close relationship between the presence of antiFHL1 autoantibodies in IIM and severe muscle pathology and poor clinical prognosis. In an effort to investigate a potential pathogenic role of immunity to FHL1 in IIM, we used an MHC class I-dependent mouse model and found that immunization with FHL1 caused a major aggravation of muscle dysfunction and increased mortality.

\section{Results}

Anti-FHL1 autoantibodies were identified using a muscle-specific cDNA library. In order to identify genes encoding putative muscle-specific autoantigens, we screened a muscle cDNA library with sera from 3 representative patients with established IIM, 1 with classical DM (patient A), 1 with cancer-associated DM (patient B), and 1 with PM and anti-histidyl-tRNA synthetase (Jo-1) antibodies (patient C) (Supplemental Table 1; supplemental material available online with this article; doi:10.1172/ JCI81031DS1). In the serum from the Jo- $1^{+}$patient, we identified several clones with cDNA inserts of the histidyl-tRNA synthetase, qualifying it as a good internal control for the methodology used. In 2 of the 3 tested sera (from patients A and B), we identified clones that had an 843-bp ORF and a predicted amino acid sequence of 281 residues with $100 \%$ identity with FHL1. As FHL1 missense mutations have been linked to congenital myopathies in earlier studies $(23,26,28,30)$, it was selected for further analysis. 
A

\section{Dysphagia}

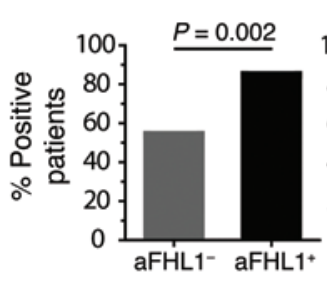

C

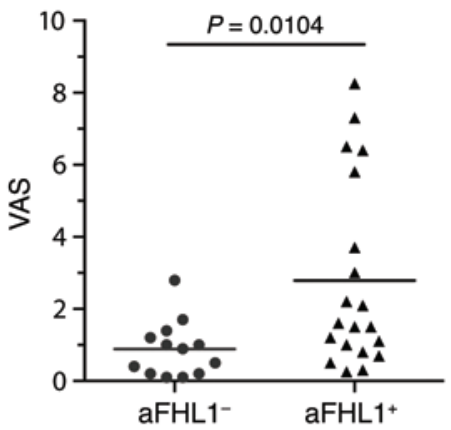

Distal muscle weakness
Clinical atrophy
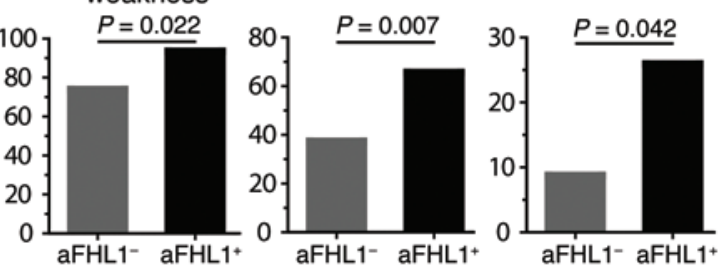

D

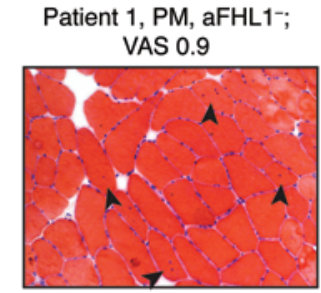

Patient 4, PM, aFHL1+; VAS 6.5

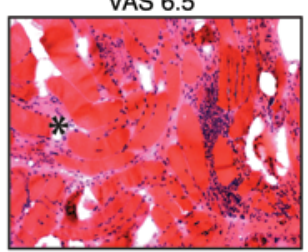

\section{Cor}

(issue

fat replacement

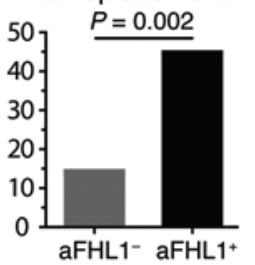

Patient 2, PM, aFHL1-

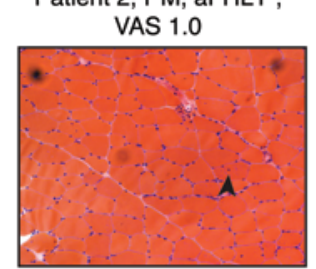

Patient 5, PM, aFHL1+; VAS 7.3

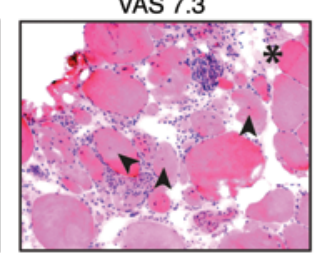

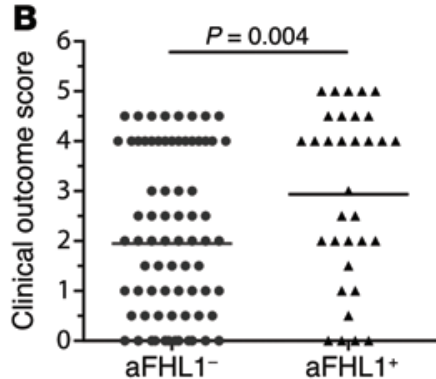

Patient 3, DM, aFHL1+; VAS 2.1

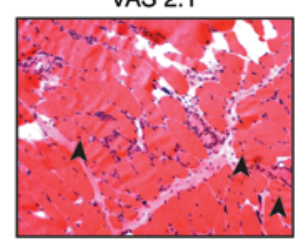

Patient 6, PM, aFHL1+; VAS 8.25

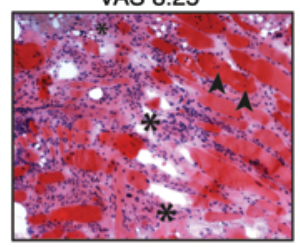

Figure 2. The presence of anti-FHL1 autoantibodies is associated with pronounced muscle damage. (A) Statistical analysis revealed that anti-FHL1 positivity was associated with dysphagia $(P=0.002$, Fisher's exact test), distal muscle weakness $(P=0.022$, Fisher's exact test), clinical atrophy $(P=0.007$, Fisher's exact test), fiber necrosis ( $P=0.042$, Fisher's exact test), and connective tissue/fat replacement $(P=0.002$, Fisher's exact test). (B) Clinical disease severity score for anti-FHL1+ compared with anti-FHL1- IIM patients. Scoring for disease severity was done by examining disease outcome determined at the patient's last clinic visit. (C) H\&E- and Gomori trichrome-stained muscle tissue sections of 20 patients with anti-FHL1 autoantibodies were examined for histopathology and compared with stained muscle tissue sections from sex-, age-, and diagnosis-matched (PM, DM, and IBM; $n=13$ ) anti-FHL1- patients. Scoring was done using a 0-10 cm VAS. (D) Representative H\&E-stained images of muscle tissue from 2 anti-FHL1- patients with low histopathological VAS scores (patients 1 and 2); 1 anti FHL1+ patient with a medium VAS score (patient 3); and 3 anti-FHL1+ patients with high VAS scores (patients 4 , 5 , and 6). Images show inflammatory infiltrates, connective tissue/fat replacement (indicated by asterisks), internal nuclei (indicated by arrows), and massive fiber size variation. Scoring in B and C was done blindly with regard to anti-FHL1 autoantibody status. Statistics for B and C were calculated by 2-tailed Mann-Whitney $U$ test; each data point represents 1 individual, and horizontal bars indicate the mean values.

FHL1 autoantibodies are specific to inflammatory myopathies. Using an ELISA able to detect IgG antibodies against FHL1 protein, we investigated sera from 141 patients with IIM, from 126 sex- and age-matched healthy controls (HCs), and from a total of 195 patients with other autoimmune diseases or neuromuscular disease (NMD). We used FHL1 coupled to maltose-binding protein $(\mathrm{MaBP})$ as an antigen and $\mathrm{MaBP}$ alone as a control. The latter showed either no or occasional reactivity, thereby demonstrating the specificity of the FHL1 ELISA. Patients with IIM showed significantly higher levels of anti-FHL1 autoantibodies compared with levels in healthy individuals (Figure $1 \mathrm{~A}, P<0.0001$ ). Examination of HCs allowed the calculation of a cutoff value by which anti-FHL1 ${ }^{-}$and anti-FHL1 ${ }^{+}$patients could be differentiated. Thus, 35 of 141 patients (24.8\%) were identified as positive for anti-FHL1 autoantibodies (Figure 1B). Of note, the cDNA library screen showing positivity for anti-FHL1 autoantibodies in patients $A$ and $B$ and negativity for these autoantibodies in patient $\mathrm{C}$ was confirmed by ELISA, indicating the reliability of these different methodologies. Dilutional linearity was demonstrated by serial dilution ELISA experiments on anti-FHL1+ and anti-FHL1 sera as well as on HC sera and allowed detection of the optimal dilution factor (Supplemental Figure 1). Anti-FHL1 positivity was subsequently confirmed by another ELISA using a recombinant His-tagged FHL1 protein (Figure 1C) and by Western blotting using an FHL1-MaBP fusion protein (Figure 1D). Western blotting also verified the absence of anti-FHL1 reactivity in HC sera and no reactivity of anti-FHL1 ${ }^{+}$sera to the MaBP protein (Figure 1D). In addition, to show reactivity of anti-FHL1 ${ }^{+}$sera to the native autoantigen, we applied sera to normal muscle tissue sections and performed immunofluorescence and confocal microscopy. As shown in Supplemental Figure 2A, staining with anti-FHL1 ${ }^{+}$ sera yielded a diffuse, homogenous cytoplasmic staining of muscle fibers of normal human muscle tissue, whereas no staining of muscle fibers was observed using HC or anti-FHL1- sera. Moreover, the staining observed upon incubation with anti-FHL1 ${ }^{+}$sera could be blocked by preincubating the serum with recombinant FHL1-MaBP protein (Supplemental Figure 2B), demonstrating reactivity of the antibodies to the native FHL1 protein in muscle fibers. The presence of anti-FHL1 autoantibodies was subsequently confirmed in an independent cohort of patients with IIM from the Czech Republic; here, 31 of 129 patients (24\%) were identified as anti-FHL1+ (Supplemental Figure 3).

To further determine the specificity of the anti-FHL1 autoantibodies, we performed ELISA to investigate their presence in other 

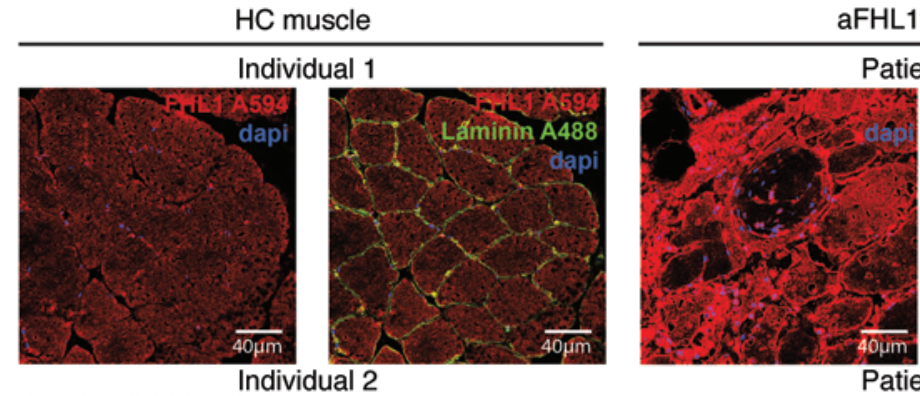

aFHL1+ myositis
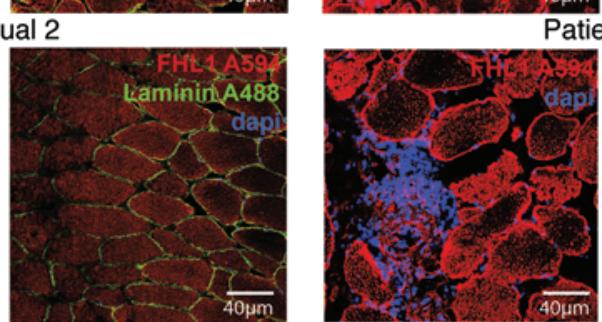

Individual 3
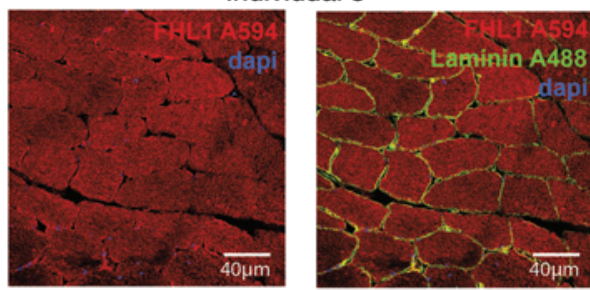

Individual 4
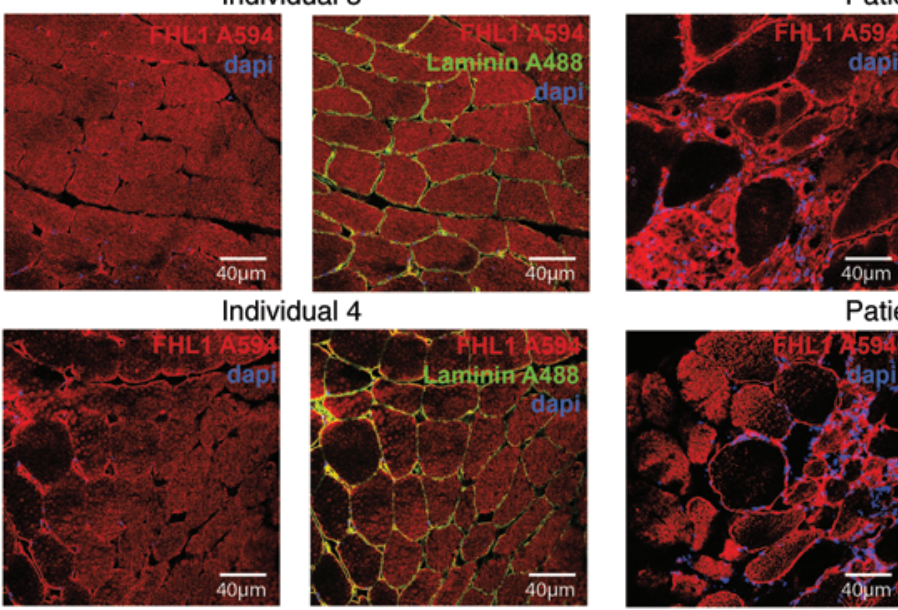

Patient 3, IBM
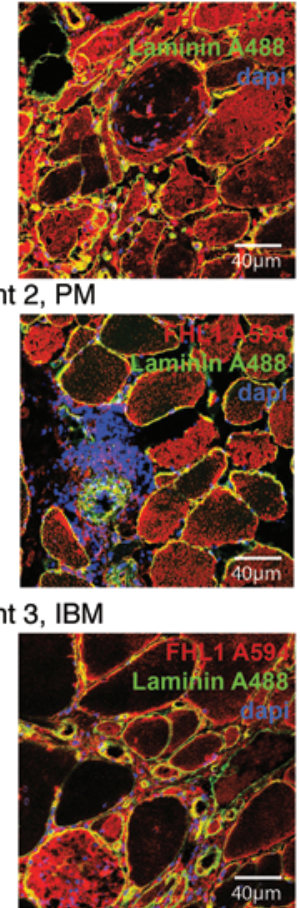

Patient 4, PM
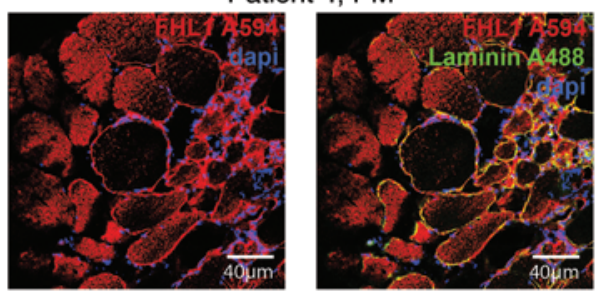

aFHL1- myositis
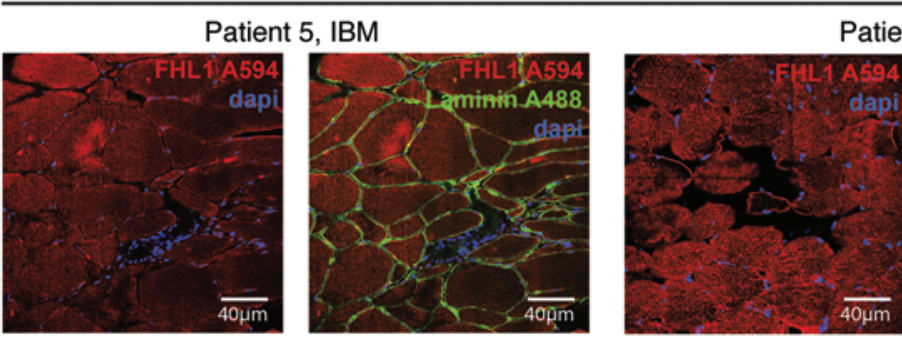

Patient 6, PM

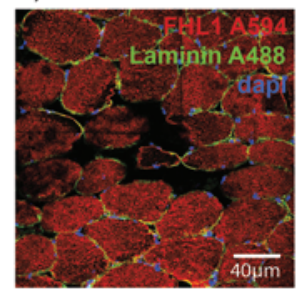

Figure 3. FHL1 protein has an altered expression pattern in muscles of antiFHL1+ IIM patients. Muscle tissue sections from HCs (left 2 panels) were compared by confocal microscopy with muscle tissue sections from anti-FHL1+ ${ }^{+}$atients (right 2 panels) and from anti-FHL1' patients (lower panels). FHL1 staining is shown in red (secondary antibody coupled to Alexa Fluor 594), and nuclei were visualized using DAPI (blue). In addition, laminin staining was done to visualize the sarcolemma (green, Alexa Fluor 488), and an overlay was done with FHL1 staining and DAPI. Scale bars: $40 \mu \mathrm{m}$. autoimmune diseases including mixed connective tissue disease (MCTD), rheumatoid arthritis (RA), primary Sjögren's syndrome (pSS), systemic lupus erythematosus (SLE), and systemic sclerosis (SSc). The majority of sera from patients with other autoimmune diseases did not show reactivity to FHL1 (Figure 1E), and only 1 of 20 (5\%) MCTD patients, 1 of 68 (1\%) RA patients, 2 of 33 (6\%) SSc patients, 1 of 36 (3\%) pSS patients, and 0 of 34 (0\%) SLE patients were anti-FHL1 ${ }^{+}$. Logistic regression analyses confirmed that anti-FHL1 autoantibodies are highly myositis specific: odds ratio $(\mathrm{OR})=14.1$ (95\% CI 4.2-47.6, $P<0.0001$ ) for IIM; OR = 1.9 (95\% CI $0.19-19.4, P=0.586)$ for MCTD; OR $=0.54$ (95\% CI $0.05-5.3, P=0.594)$ for $\mathrm{RA} ; \mathrm{OR}=2.3$ (95\% CI 0.37-14.7, $P=0.368$ ) for SSc; and OR = 1.1 (95\% CI 0.11-11.2, $P=0.923$ ) for pSS, when adjusting for age and sex and using HCs as a reference. The
ELISA results yielded a $25 \%$ sensitivity, a $97 \%$ specificity, and an $80 \%$ positive predictive value for diagnosing myositis using antiFHL1 autoantibodies.

In addition, in patients with noninflammatory NMD with muscle weakness, we could not detect any anti-FHL1 reactivity (Figure $1 \mathrm{E})$, indicating that the autoantibodies are not formed merely as a consequence of excessive destruction of muscle tissue.

The HLA DRB1*03/ ${ }^{*} 13$ genotype is more frequent in anti-FHL1 ${ }^{+}$ patients. To elucidate a potential immunogenetic contribution, we compared the frequency of HLA-DRB1 haplotypes in anti$\mathrm{FHL1}^{+}$and anti-FHL1 ${ }^{-}$patients. We found that the combination of HLA-DRB $1{ }^{*} 03 /{ }^{*} 13$ haplotypes was more frequent in anti$\mathrm{FHL1}^{+}$patients than in anti-FHL1 ${ }^{-}$patients; 7 of 33 anti-FHL1 ${ }^{+}$ patients $(21.2 \%)$ were found to have this genotype compared 

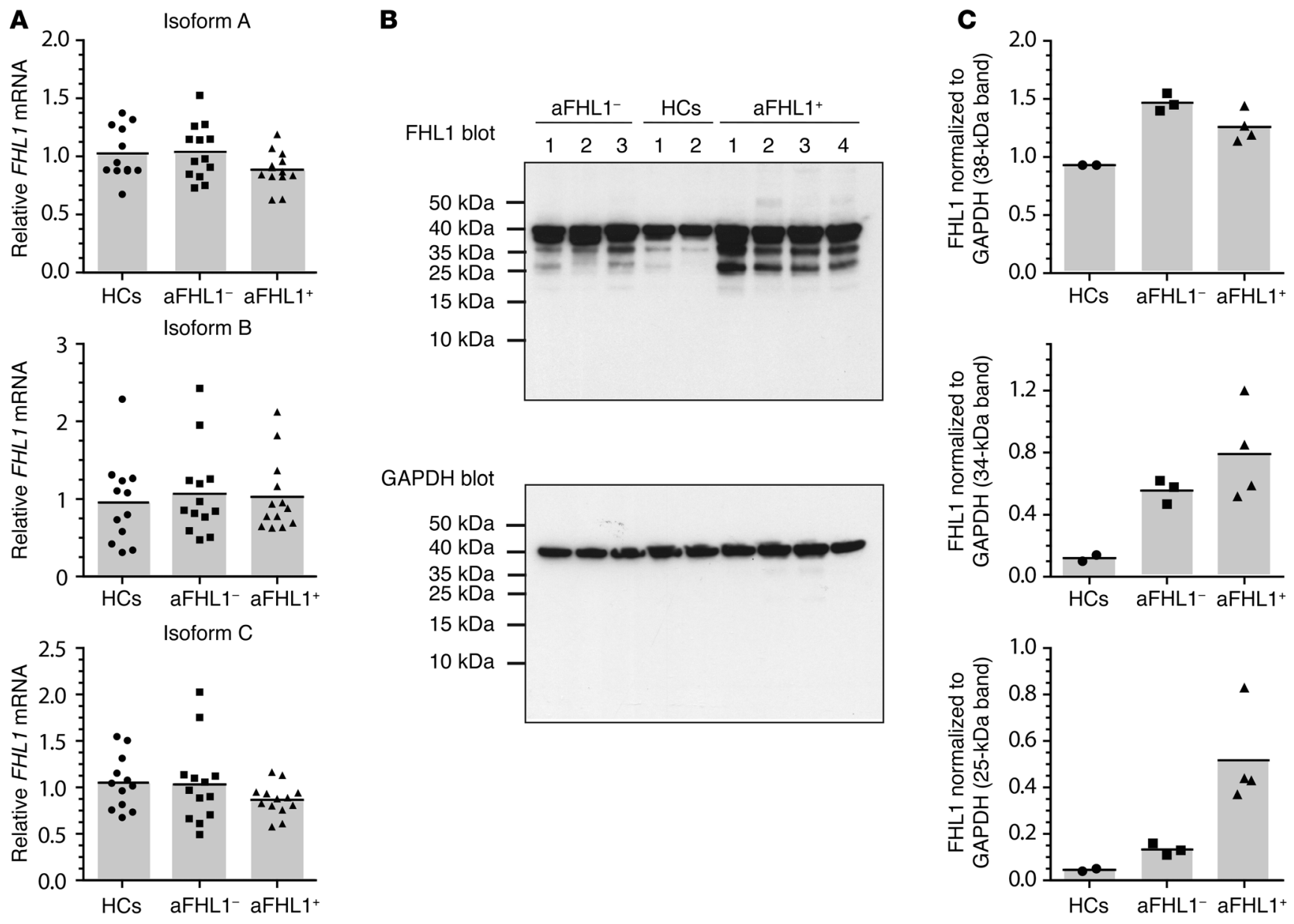

Figure 4. A higher amount of lower-molecular-weight protein bands can be detected in anti-FHL1+ muscle biopsy lysates. (A) mRNA was extracted from muscle biopsies from anti-FHL1+ $(n=13)$ and anti-FHL1- $(n=13)$ patients as well as from HCs $(n=12)$ and transcribed into cDNA. FHL1 mRNA expression was analyzed by TaqMan PCR using specific primers for amplification of the A, B, and C isoforms. Each data point represents 1 individual, and horizontal bars indicate the mean values. (B) Protein lysates were generated from muscle biopsy material from anti-FHL1+ $(n=4)$ and anti-FHL1- $(n=3)$ patients as well as from healthy muscle tissue $(n=2)$ and immunoblotted with commercially available anti-FHL1 antibody. GAPDH was used as a loading control for immunoblotting. (C) The 3 major bands detected by immunoblotting were quantified using Quantity One 1-D Analysis software and normalized to the GAPDH loading control by calculating as follows: FHL1 band /GAPDH band

with 6 of 88 anti-FHL1 ${ }^{-}$patients $(6.8 \% ; P=0.02)$ (Supplemental Figure 4, Supplemental Table 2).

Anti-FHL1 autoantibodies are associated with severe muscle pathology. Long-term clinical follow-up data were available for 132 of 141 (94\%) patients with IIM (anti-FHL1 ${ }^{+}, n=33$; anti-FHL1 ${ }^{-}, n=$ 99) (Supplemental Table 3). FHL1 autoantibodies were detectable in all 3 IIM subtypes; 19 of 33 anti-FHL1 ${ }^{+}$patients were diagnosed with PM (58\%), 10 with DM (30\%), 1 with juvenile DM (3\%), and 3 of 33 patients with IBM (9\%), reflecting the frequencies of the subtypes in the investigated myositis cohort (see Supplemental Table 3). Anti-FHL1 ${ }^{+}$patients developed dysphagia more often than did anti-FHL1 ${ }^{-}$patients (28 of 33 [85\%] anti-FHL1 ${ }^{+}$compared with 52 of 96 [54\%] anti-FHL1 ${ }^{-}$patients, $P=0.002$ ) (Figure 2A and Supplemental Table 4). We also found significant associations between the presence of anti-FHL1 autoantibodies and distal muscle weakness $(P=0.022)$, clinical muscle atrophy $(P=0.007)$, and vasculitis $(P=0.008)$ (Figure 2A and Supplemental Table 4$)$. To further examine the associations between the presence of antiFHL1 autoantibodies and clinical muscle pathology, we developed a scoring system with a focus on muscle involvement determined at the patient's most recent medical examination. Anti-FHL1 ${ }^{+}$ patients had a worse clinical outcome score compared with that for anti-FHL1 ${ }^{-}$patients, and patients with the worst possible disease score were found in the anti-FHL1 $1^{+}$group (Figure 2B). These patients were characterized by a high degree of muscle weakness or complete loss of ambulation and a remarkably progressive and therapy-resistant disease history.

We also found significant associations between the presence of anti-FHL1 autoantibodies and muscle biopsy features including fiber necrosis $(P=0.042)$ and connective tissue replacement of muscle tissue $(P=0.002)$, as evidenced by examination of the first available muscle biopsy specimen (Figure 2A and Supplemental Table 4). The observed association with distinct muscle biopsy features in anti-FHL1+ ${ }^{+}$patients was further supported when all available H\&E- and Gomori trichrome-stained muscle tissue sections from 20 anti-FHL1 ${ }^{+}$patients and 13 sex-matched, age-matched, and diagnosis-matched (PM, DM, and IBM) anti-FHL1- patients were scored for severity according to a visual analog scale (VAS) 
A

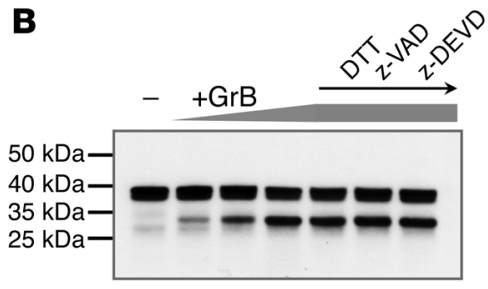

C His tag D Recombinant E MaBP-FHL1 fusion protein FHL1 FHL1

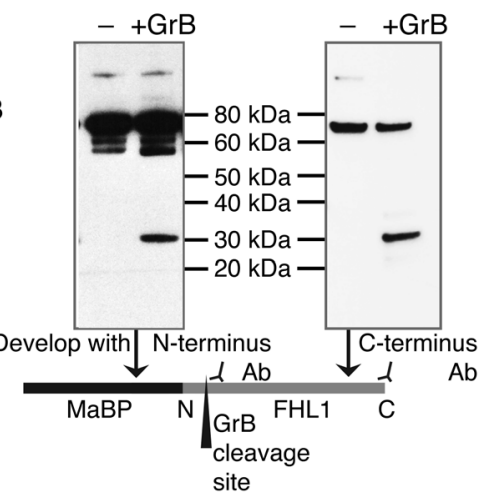

$\mathbf{F}$

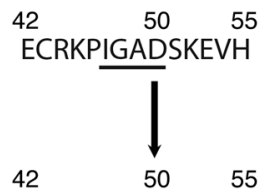

ECRKPIGAASKEVH
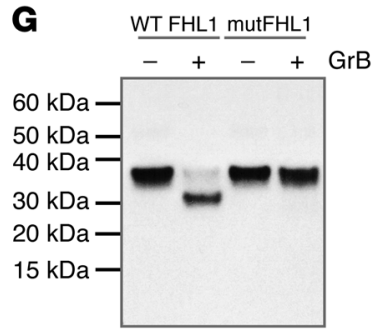

(31). Patients with autoantibodies against FHL1 had a mean muscle biopsy severity VAS score of $2.8 \mathrm{~cm}$, whereas anti-FHL1- patients had a VAS score of $0.9 \mathrm{~cm}$ (Figure 2C). As shown in the representative tissue sections in Figure 2D, anti-FHL1 ${ }^{+}$patients with high VAS scores, as compared with anti-FHL1- patients with low VAS scores and an anti-FHL1 $1^{+}$patient with a medium VAS score, had more connective tissue and fat replacement (Figure 2D, asterisks), internal nuclei (Figure 2D, arrows), variation of fiber size, and more frequently pronounced inflammatory infiltrates. The overall histopathology score also revealed that patients with the highest possible VAS score (over $6.0 \mathrm{~cm}$ ) were found in the anti-FHL1+ group, further confirming a severe muscle histopathology.

To further test the stability of the anti-FHL1 autoantibody response and its sensitivity to treatment over time in either severely affected patients or patients with mild disease, we performed ELISAs on longitudinal serum samples from representative patients (Supplemental Figure 5). For the analyzed patients with progressive disease refractory to therapeutic intervention and characterized by a poor outcome score (determined in Figure 2B), anti-FHL1 autoantibody levels were constantly above the cutoff levels of healthy individuals (Supplemental Figure 5A). Selected patients with good response to therapy were anti-FHL1 ${ }^{+}$only occasionally (Supplemental Figure 5B), and anti-FHL1- patients appeared negative over the entire analysis period (Supplemental Figure 5C). We could not detect any general influence of treatment on serum levels of anti-FHL1 autoantibodies, and anti-FHL1 autoantibody levels did not correlate with variations in serum levels of creatine kinase (CK) (Supplemental Table 5).

Figure 5. FHL1 is a target of granzyme B. (A) Cleavage of FHL1 by granzyme $B$ was demonstrated by performing a cleavage assay using skeletal muscle cell lysates and increasing concentrations of recombinant granzyme B (50, 100, and $250 \mathrm{U})$ and (B), as indicated, in lanes 5-7, DTT (5 mM), z-VAD (500 nM), or z-DEVD (500 nM) was added. The results for the cleavage assay are representative of 5 independent experiments. In addition, recombinant His-tagged FHL1 (C), commercially available FHL1 (OriGene) (D), and FHL1-MaBP fusion protein (E) were cleaved with granzyme B. For the latter, the immunoblot was developed with either an antibody binding to the $\mathrm{N}$-terminus or an antibody binding to the C-terminus to identify the location of the cleavage site. (F) The granzyme B cleavage site in FHL1 was predicted to be IGAD, with $D$ at amino acid position 50 . (G) Using sitedirected mutagenesis, the cleavage site was mutated and IVTT-expressed WT or mutated FHL1 (mutFHL1) was incubated with granzyme B or left untreated and analyzed by immunoblotting.

We found no associations between anti-FHL1 autoantibodies and any of the laboratory measurements (Supplemental Table 5), nor did we detect the presence of other autoantibodies including the muscle-specific autoantibody $\mathrm{cN}-1 \mathrm{~A}$ (ref. 7 and Supplemental Table 6). Our analysis of the distribution of the $P$ values reported in Supplemental Tables 4 and 5 revealed a proportion of less than $10 \%$ of low $P$ values that was significantly higher $(P<0.0001)$ than what would be expected by chance (Supplemental Figure 6). These associations indicate a link between positivity for anti-FHL1 autoantibodies and disease severity, particularly that affecting skeletal muscle.

FHL1 shows an altered expression pattern in muscles of anti-FHL1+ patients. To gain insights into mechanisms of muscle damage related to anti-FHL1+ inflammatory myopathies, we used immunofluorescence staining with a commercial anti-FHL1 antibody and confocal microscopy to examine FHL1 protein expression in muscle tissue from patients with anti-FHL1 ${ }^{+}$myositis compared with that from healthy individuals and anti-FHL1' ${ }^{-}$patients. Healthy muscle tissue (Figure 3, left 2 panels) and muscle tissue from antiFHL1- patients (Figure 3, bottom panels) showed a homogenous expression pattern of FHL1 that was mainly sarcoplasmic and distributed uniformly along the muscle fiber. In patients with anti$\mathrm{FHL1}^{+}$myositis, the pattern was strikingly different; some fibers had focal accumulations of FHL1, whereas others had almost no FHL1 expression (Figure 3, right 2 panels). In addition, we observed colocalization of FHL1 with laminin in the sarcolemma, which was less obvious in muscle tissue from HCs. Specificity of the staining was confirmed by using appropriate isotype controls (Supplemental Figure 7A) and by blocking FHL1 staining with recombinant FHL1 (Supplemental Figure 7B).

As FHL1 can undergo alternative splicing and different isoforms have been described (20), we performed mRNA expression analysis of the 3 major $F H L 1$ isoforms (isoforms A, B, and C). All 3 isoforms were expressed similarly in HC, anti-FHL1', and anti-FHL1 ${ }^{+}$muscle tissue lysates (Figure $4 \mathrm{~A}$ ), with a tendency toward lower expression levels of isoform A in anti- $\mathrm{FHL}_{1}{ }^{+}$versus anti-FHL1- lysates. To investigate whether FHL1 mRNA expression is reflected by protein expression, we performed Western blot analysis of muscle tissue lysates. In HC muscle tissue lysates, 1 major band at around $38 \mathrm{kDa}$ was detected (Figure 4B). In contrast, Western blot analysis of muscle tissue lysates from anti-FHL1 ${ }^{+}$patients showed strong expression of 2 additional bands at 34 and $25 \mathrm{kDa}$ (Figure 4B). All 3 bands contained FHL1, 
A
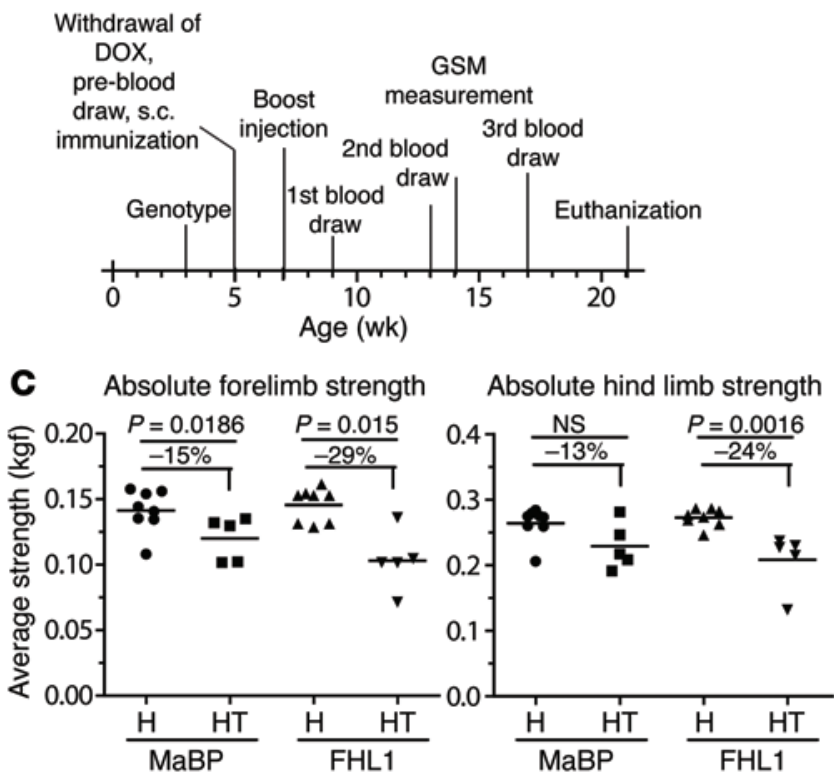

$\mathbf{F}$
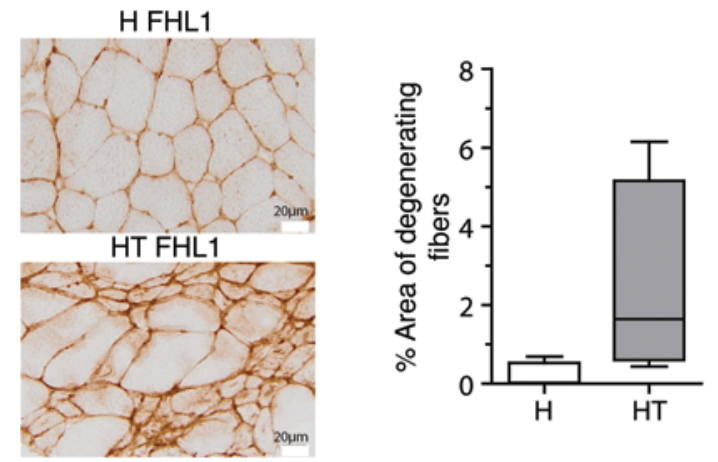

B

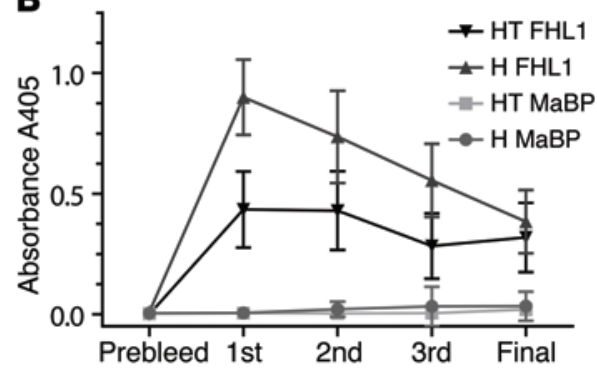

$\mathbf{E}$
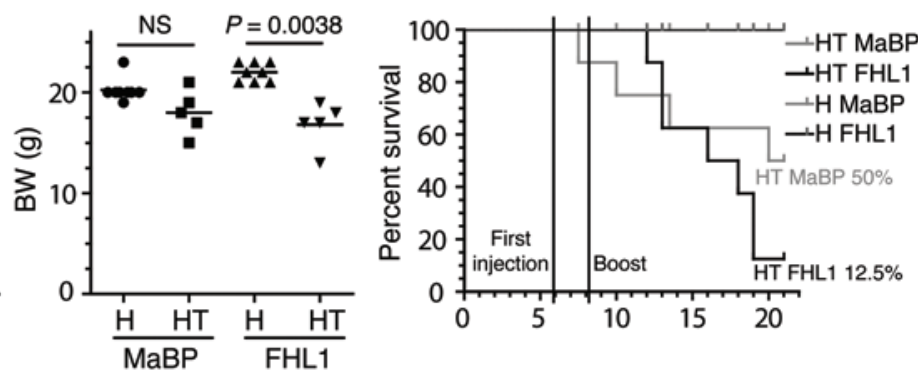

G
Gzmb

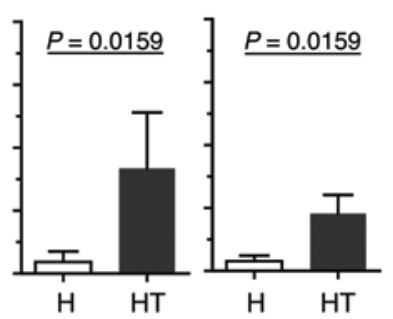

Figure 6. Immunization of HT mice with FHL1 aggravates muscle pathology. At the time points indicated in the animal experimental scheme (A), serum was taken and analyzed by ELISA for the presence of anti-FHL1 autoantibodies, with recombinant His-tagged FHL1 as an antigen (B). DOX, doxycycline; GSM, grip strength measurement. At the age of 16 weeks, 9 weeks after the mice were immunized, forelimbs and hind limb grip strength was measured using a grip strength meter (C), and BW was determined (D). H MaBP mice and H FHL1 mice, $n=8$; HT MaBP mice and HT FHL1 mice, $n=5$. $P=0.0038,2$-tailed Mann-Whitney $U$ test. (E) The hazard ratio of the Kaplan-Meier survival plot is 2.2, indicating that mice in the HT-FHL1 group died 2.2 times faster than did mice in the matching MaBP control group. (F) Muscle injury was determined by staining of IgM depositions in HT FHL1 $(n=5)$ versus H FHL1 ( $n=4)$ mice. Scale bars: $20 \mu \mathrm{m}$. (G) Hind-limb muscle tissue from HT $(n=5)$ and H mice $(n=4)$ immunized with FHL1 was analyzed by qPCR for relative mRNA expression of $C d 4$, Cd8a, Gzmb, and Prf1. P values in $\mathbf{G}$ were calculated by a 2-tailed Mann-Whitney $U$ test.

as shown by gel extraction and subsequent mass spectrometric analysis (Supplemental Figure 8). Quantification of intensity and normalization to the housekeeping gene GAPDH for these 3 bands revealed that expression of the $38-\mathrm{kDa}$ band was comparable between muscle lysates from HCs and from patients with myositis, with a tendency toward lower expression in anti-FHL1 ${ }^{+}$ versus anti-FHL1 ${ }^{-}$patients (Figure $4 \mathrm{C}$ ). In contrast, expression intensity of the 34- and 25-kDa bands consistently increased in muscle tissue lysates from both HCs and anti-FHL1- patients and reached a maximum in muscle tissue lysates from anti-FHL1 ${ }^{+}$ patients (Figure 4C).

Thus, muscle tissue lysates from anti-FHL1 ${ }^{+}$patients showed a distinct protein expression pattern, as demonstrated by both immunofluorescence confocal microscopy and Western blot analysis, suggesting that the protein might be subject to degradation in a subset of patients with myositis and that this degradation is most pronounced in anti-FHL1 ${ }^{+}$patients.
FHL1 is susceptible to cleavage by granzyme B. Cleavage of autoantigens mediated by the cytotoxic protease granzyme $B$ is a frequent feature of systemic autoimmune diseases and is believed to contribute to the initiation and propagation of autoimmunity (32). We therefore addressed the question of whether endogenous FHL1 might be cleaved by granzyme B by performing in vitro cleavage assays with human skeletal muscle cell lysates. Indeed, we observed a dose-dependent fragmentation of FHL1 in the presence of increasing concentrations of granzyme B (Figure $5 \mathrm{~A}$ ). To eliminate the role of endogenous muscle caspases in the cleavage of FHL1, we used different inhibitors to inactivate caspase activity in the muscle lysate. Interestingly, the presence of $\mathrm{z}-\mathrm{VAD}$ and $\mathrm{z}$-DEVD could not prevent granzyme B-mediated cleavage of FHL1 (Figure 5B), demonstrating that muscle FHL1 is a direct substrate of granzyme $\mathrm{B}$, with a resulting cleavage fragment of $30 \mathrm{kDa}$. Direct cleavage of FHL1 by granzyme B was further confirmed using recombinant proteins; all proteins tested, 
including His-tagged FHL1 (Figure 5C), commercially available recombinant FHL1 (Figure 5D), and FHL1-MaBP fusion protein (Figure 5E), were cleaved, and the 30-kDa cleavage fragment was detected. As this fragment was detectable with both an antibody binding to the $\mathrm{N}$-terminus and another antibody binding to the $\mathrm{C}$-terminus of the protein (under the conditions used in Figure $5 \mathrm{E}$ ), we concluded that the cleavage site is located in the N-terminal portion (Figure $5 \mathrm{E}$, scheme). Tetrapeptide substrate specificity of human granzyme B has been determined previously $(33,34)$. This specificity and the fragment sizes generated by granzyme $B$ cleavage were used to predict that the cleavage site in FHL1 is the tetrapeptide IGAD with $\mathrm{P} 1$ aspartic acid at amino acid position 50 (Figure 5F). The identified cleavage site was confirmed by sitedirected mutagenesis of the relevant $\mathrm{P} 1$ aspartic acid to alanine $\left(D_{50} \rightarrow A_{50}\right)$, leading to resistance of FHL1 protein to cleavage by granzyme B (Figure $5 G$ ).

Anti-FHL1 immunity in MHC class I transgenic mice aggravates muscle weakness. To further analyze the role of FHL1 in IIM pathogenesis, we used an IIM mouse model. As previously demonstrated, upregulation of MHC class I in skeletal muscle causes muscle inflammation and weakness in double-transgenic mice (referred to herein as HT mice), whereas single-transgenic littermates (referred to herein as $\mathrm{H}$ mice) do not develop IIM symptoms (35). To study the impact of an immune response formed against FHL1, we immunized myositis-susceptible HT and control $\mathrm{H}$ mice with either FHL1-MaBP protein or MaBP alone. Blood was collected at several time points after immunization (Figure 6A), and sera were analyzed by ELISA for FHL1 reactivity. We detected anti-FHL1 autoantibodies in both $\mathrm{H}$ and HT mice immunized with FHL1-MaBP (Figure 6B). H mice receiving FHL1 fusion protein or MaBP were unaffected during the experimental period. Nine weeks after immunization, we detected an increased weakness of forelimbs and hind limbs in both $\mathrm{HT}$ groups compared with the $\mathrm{H}$ mice. In addition, a more pronounced weakness was observed in the FHL1-immunized HT group compared with the MaBP control-immunized mice (Figure $6 \mathrm{C}$ ). On average, the forelimbs of HT mice immunized with MaBP lost $15 \%$ of their grip strength compared with the healthy H mice, while FHL1-immunized HT mice lost 29\% of their grip strength compared with the healthy $\mathrm{H}$ mice. For the hind limbs, grip strength was reduced by $13 \%$ in the MaBP-immunized HT mice compared with the $\mathrm{H}$ mice versus a $24 \%$ grip strength reduction observed in the FHL1-immunized HT mice (Figure 6C). BW measurement revealed no statistically significant difference in the MaBP control groups. However, weight loss in the group of FHL1-immunized HT mice was pronounced and statistically significantly increased compared with that of the FHL1-immunized $\mathrm{H}$ mice (Figure 6D). The survival rate for the FHL1-immunized HT group was $12.5 \%$ (1 of 8 mice in total survived until the end of the experiment), whereas the survival rate for the HT MaBP group was $50 \%$ (Figure $6 \mathrm{E}$ ). The hazard ratio was determined to be 2.20, indicating that mice in the HT FHL1-MaBP group died at 2.2 times the rate of mice in the control HT MaBP group for the same time period.

As expected, FHL1-immunized HT mice showed prominent muscle cell damage, demonstrated by IgM depositions in muscle tissue (Figure 6F). In addition, we observed increased expression levels of $C d 4$ and $C d 8 a$ as well as Gzmb and Prf1 in the hind-limb muscle tissue of FHL1-immunized HT mice (Figure 6G), indicating the presence of $\mathrm{T}$ cell infiltrates.

\section{Discussion}

Our study identifies what we believe to be a novel and musclespecific autoantigen, FHL1, in a subset of IIM patients characterized by severe skeletal muscle involvement. Strikingly, mutations of the encoding FHL1 gene are associated with severe inherited myopathy $(23,26,28,30)$. Immunization of myositis-prone mice with FHL1 aggravated muscle weakness and caused increased mortality. Together, these data suggest that we have identified a new variant of severe myopathy characterized by the presence of anti-FHL1 autoantibodies that may represent a new prognostic biomarker for this disease variant.

Our current approach using a muscle-specific expression library for detecting new muscle-specific autoantigens has not, to our knowledge, been used previously to identify autoantibody targets in IIM. The present study showing that the FHL1 antigen is a muscle-specific target in IIM therefore illustrates the power of this method and suggests that its application may identify additional autoantigens.

Which factors could account for FHL1 becoming an autoantigen that consequently drives the development of anti-FHL1 autoantibodies? Multiple studies have demonstrated that the majority of autoantigens targeted in systemic autoimmune diseases are substrates of granzymes, in particular granzyme B (32, 36-40), a serine protease found in the cytoplasmic granules of cytotoxic T lymphocytes and NK cells. Granzyme B-mediated cleavage of autoantigens can lead to the generation of neo-epitopes and thus promote initiation of immune responses (32). It can also influence protein function as well as redistribution within the cell $(36,37,40,41)$. Our finding that FHL1 is a target of granzyme $B$ in vitro suggests a scenario that may also be operating in vivo in this subset of myositis and could explain the lower-molecular-weight fragments of FHL1 in the muscle detected by Western blotting (bands migrated at 34 and $25 \mathrm{kDa}$ ). Thus, susceptibility to granzyme cleavage could drive the exposure of novel FHL1 epitopes and lead to a subsequent break of tolerance and initiation of autoimmunity (Supplemental Figure 9). Here, 2 scenarios are possible: (a) cleavage of FHL1 might be a primary event during tissue damage, triggering a secondary immunity to FHL1, or (b) antibodies might first be generated against intact or cleaved FHL1, and, consequently, anti-FHL1 immunity becomes a driving feature and initiating event of this subset of IIM. We cannot at present distinguish between these 2 possibilities since we do not have access to the necessary pre-disease sera. However, considering the results of our longitudinal serum samples from patients with progressive disease, we can conclude that immunity to FHL1 appears at early time points of the disease and is characterized by remarkable stability and persistence, suggesting that it probably contributes to and aggravates IIM.

In addition, granzyme B cleavage or other mechanisms like posttranslational modifications, e.g., by oxidation (42), could induce instability of the secondary structure of FHL1 protein, similar to the events observed in genetic FHL1-related myopathies, which, consequently, might result in loss of protein functionality. Given the 
role of FHL1 as a critical modulator of muscle mass and strength enhancement (12), loss of FHL1 functionality could account for muscle degeneration and severe muscle pathology. Importantly, in muscle tissue of IIM patients with anti-FHL1 autoantibodies, FHL1 showed an altered expression pattern with focal accumulations. The pattern observed in anti-FHL1 $1^{+}$patients is remarkably similar to that caused by mutations of FHL1 in patients suffering from the genetic FHL1-related myopathy RBM, in which FHL1 appears to be progressively incorporated into intracytoplasmic inclusions (23).

The consequence of an autoimmune response to FHL1 for the pathogenesis of inflammatory myopathies was examined in IIM patients and in an MHC class I-dependent mouse model for myositis (35). In humans, the association between the presence of anti-FHL1 antibodies and myofiber damage is compatible with both a model in which immunity develops first and has secondary effects on muscle cells and a model in which neo-epitopes are primarily found in the muscle, and immunity occurs as a result of neo-epitope exposure and local activation of antigen-presenting cells. In addition, the $H L A D R B 1^{*} 03 /{ }^{*} 13$ genotype was found to be more frequent in anti-FHL1 ${ }^{+}$patients, suggesting an involvement of classical MHC class II-dependent antigen presentation and $\mathrm{CD}^{+} \mathrm{T}$ cell help in the formation of anti-FHL1 autoantibodies. $H L A$ alleles are known to be strongly associated with the development of myositis-specific antibodies, as demonstrated for different specificities (43-45), and a genome-wide association study in DM confirms the importance of $H L A$ as a genetic contributor to IIM (46). The mouse experiment indicates that immunity to FHL1 may indeed contribute to IIM pathology, but so far only in mice that already have a mild form of the disease. This scenario is of course also possible in the human setting. In addition to autoantibodies detectable in the circulation, the animal model also revealed indications for $\mathrm{T}$ cell infiltrates present in the inflamed tissue that likely contribute to tissue injury and pathogenesis. Cytotoxic $\mathrm{CD}^{+} \mathrm{T}$ cells are of particular importance in IIM pathogenesis and were shown to be clonally expanded and to invade MHC class I-expressing muscle fibers (47-50). Whether antiFHL1 autoantibodies themselves harbor pathogenic capacity and drive tissue destruction and fibrosis in addition to cell-mediated autoimmunity remains to be determined by future investigations.

Importantly, however, the newly identified anti-FHL1 antibodies may function as important biomarkers for a subset of severe inflammatory myopathy. Of particular interest in this context is the high frequency of patients with dysphagia, which has been previously described to be associated with high disease severity in IIM $(51,52)$, and an association with pronounced skeletal muscle damage.

In conclusion, we have identified and characterized what is, to our knowledge, a novel muscle-specific autoantigen in a subset of patients classified as having IIM with severe skeletal muscle involvement and a risk of poor prognosis. Thus, our study indicates a role of FHL1 in the pathogenesis of a subset of IIM, emphasizing the need for careful characterization of IIM disease subsets to improve our understanding of the molecular pathways. Further investigations of FHL1 and its corresponding autoantibodies in IIM may shed light on the possible mechanisms of development of autoimmunity in inflammatory muscle disease. In particular, a detailed characterization of the mechanisms that account for
FHL1 becoming a central autoantigen in IIM in vivo, including the identification of antigenic FHL1 epitopes, is still needed. Another limitation is the small sample size in this study, in particular for the analysis of possible overrepresentations of a combination of HLA haplotypes. An extensive replication of the statistical analysis of the clinical parameters and genetic associations in a multicenter cohort will thus be an important future objective. Since FHL1 itself was described to positively modulate muscle growth and strength enhancement (12), a better understanding of the physiological and pathological functions associated with FHL1, its mutations, and autoimmunity may also lead to novel therapeutic approaches for inflammatory myopathies.

\section{Methods}

Patients and clinical data. For ELISAs, sera from patients with IIM ( $n=141)$, followed at the Rheumatology Unit of Karolinska University Hospital, were compared with sera from sex- and age-matched HCs $(n=126)$, as well as sera from patients from the same clinic diagnosed with MCTD $(n=19)$, RA $(n=67)$, pSS $(n=35)$, SLE $(n=33)$, SSc $(n=32)$, and sera from patients with noninflammatory_neuromuscular diseases $(n=9)$ followed at the Neurology Clinic of Linköping University Hospital in Linköping, Sweden (for RA, pSS, SLE, and SSc, patients were sex- and age-matched with anti-FHL1+ patients). The neuromuscular diseases represented different noninflammatory, weakness-causing myopathies (3 patients with limb girdle muscular dystrophy [LGMD], 3 patients with myotonic dystrophy type 1 [DM1], 1 patient with congenital myasthenia, 1 patient with mitochondrial myopathy, and 1 patient with spinal muscular atrophy type 3 [SMA3]). The diagnoses were supported by muscle biopsies, electromyograms (EMGs), and the clinical features in each case. Hereditary myopathy was evident in 6 of 9 cases.

In addition, sera from a myositis cohort $(n=129)$ from the Institute of Rheumatology and the Department of Rheumatology of Charles University in Prague, were examined and compared with sera from HCs $(n=81)$. Myositis patients fulfilled the diagnostic criteria for definite or probable PM or DM $(53,54)$ or IBM $(55)$. To characterize FHL1 as an autoantigen more in detail, we analyzed muscle biopsies from 17 IIM patients (PM, $n=13$; DM, $n=1$; IBM, $n=3$ ) from the Swedish cohort and from $4 \mathrm{HCs}$ by microscopy and/or Western blotting.

For 132 of 141 patients in the Swedish IIM cohort (anti-FHL1', $n=33$; anti-FHL1 ${ }^{-}, n=99$ ), detailed clinical and laboratory data from patient records at Karolinska University Hospital and from the Webbased SweMyoNet and Euromyositis registries were available. We retrieved and analyzed the following parameters: HLA-DRB1 haplotypes (Supplemental Table 2); descriptive characteristics including sex, diagnosis, demographic data, and disease duration (Supplemental Table 3); clinical muscle features, skin manifestations, extramuscular involvement, and muscle biopsy features (Supplemental Table 4); clinical laboratory measurements (Supplemental Table 5); and autoantibody status (Supplemental Table 6). To estimate the severity of clinical muscle involvement, we developed the following scoring system, considering (a) the overall outcome including the physician's myositis damage index (MDI) VAS score (established by the International Myositis Assessment and Clinical Studies Group [IMACS]) (56) retrieved from the SweMyoNet registry; (b) the overall disease severity; and (c) the response to treatment: remission without treatment $=0$; remission without treatment with an MDI VAS score greater than $2.0=0.5$; remis- 
sion with treatment $=1$; low disease activity with treatment $=2$; moderate disease activity $=3$; refractory, progressive disease, persistent, therapy resistant $=4$; an MDI VAS score greater than 5.0 but less than 7.0 $=4.5$; and an MDI VAS score greater than $7.0=5$, respectively. Scoring was done for the last recorded visit by the treating physician who was blinded with regard to the presence of anti-FHL1 autoantibodies.

Screening of a muscle-specific library. Putative autoantigens were initially identified using a commercially available muscle cDNA library (Uni-ZAP XR Premade Library; Statagene, Agilent Technologies). The library was screened using sera from 3 representative patients with adult DM or PM $(53,54)$, selected according to the following inclusion criteria: patient A represented a classical case of DM; patient $\mathrm{B}$ was representative of a diagnosis of DM with an associated malignancy, since patients with paraneoplastic malignancies often develop autoantibodies $(57,58)$; and patient $\mathrm{C}$ was selected because he was considered to have typical PM and had anti-Jo-1 antibodies. Anti-Jo-1 autoantibody positivity was used as a criterion to support the accuracy of PM diagnosis, which should be clearly distinct from a noninflammatory myopathy. In addition, the presence of this well-known myositis-specific autoantibody was used as an internal control for the cDNA library screening methodology. IBM was not considered in the initial screen, as the role of the immune system in the pathogenesis of IBM is still debatable, and the pathology of this IIM subtype is characterized by a complex interplay of inflammatory and degenerative mechanisms $(59,60)$. After 4 immunoscreenings using sera from the 3 selected patients, clones were isolated and subjected to PCR amplification as well as DNA sequencing and basic local alignment search tool (BLAST) searches of the Human Genome database (http://www. ncbi.nlm.nih.gov/BLAST/).

Cloning and expression of recombinant proteins. Human FHL1 coding DNA sequence (CDS) was amplified from the human FHL1 cDNA ORF clone (transcript variant 2; NM_001449; OriGene) introducing EcoRI (5'-CAACAAGAATTCATGGCGGAGAAGTTTGACTG-3') and SalI (5'-CAACAAGTCGACTTACAGCTTTTTGGCACAG-3') restriction sites and ligated into the multiple cloning site of the pTNT vector (Promega). Using the pTNT vector, the FHL1 ORF was subcloned into either pMAL-cRI (New England BioLabs) or pET-28a (Novogen) expression vectors and proteins with an MaBP tag and a His tag, respectively, were expressed in BL21 E. coli cells. WT MaBP was used as a control. Full-length proteins were purified on amylose columns (for MaBP fusion proteins; New England BioLabs) and Ni-NTA columns (for His-tagged proteins; Pierce, Thermo Fisher Scientific), respectively, according to the manufacturers' instructions. In some experiments, a commercially available recombinant FHL1 protein (OriGene, transcript variant 2, GenBank accession number NM_001449) was used.

ELISA for detection of anti-FHL1 antibodies. ELISA was performed as previously described (61). Briefly, 96-well MaxiSorb plates (Nunc) were coated with $1 \mu \mathrm{g}$ recombinant FHL1-MaBP or Histagged FHL1 full-length protein per well diluted in carbonate buffer, $\mathrm{pH} 9.6$, overnight at $4^{\circ} \mathrm{C}$. Human sera were measured at a dilution of 1:500; mouse sera were diluted at 1:1,000. Absorbance was measured at $405 \mathrm{~nm}\left(\mathrm{~A}_{405}\right)$. For ELISAs with the FHL1-MaBP fusion protein, all sera were tested for reactivity to MaBP alone. In general, the OD values for MaBP-only ELISAs were less than 0.2. Absorbance obtained from FHL1-MaBP ELISAs was normalized to reactivity to MaBP alone by subtracting MaBP-A ${ }_{405}$ from FHL1-MaBP-A ${ }_{405}$. Dilutional linearity was ensured by performing serial dilution ELISAs in
1:2 dilution steps. A cutoff value discriminating between anti-FHL1+ and anti-FHL1- patients was defined as the mean $\mathrm{A}_{405}$ plus 2 times the $\mathrm{SD}$ of the $\mathrm{HC}$ value. For representative patients, longitudinal serum samples were measured. All samples were measured in duplicate, and anti-FHL1 ${ }^{+}$and anti-FHL1 ${ }^{-}$sera measurements on every plate ensured functionality of the assay.

Immunoblot analysis. Immunoblotting was performed with a monoclonal anti-FHL1 antibody (catalog ab76912; Abcam), mouse anti-GAPDH mAb (catalog 398600; Invitrogen), or rabbit antiGAPDH mAb (catalog 14C10; Cell Signaling Technology), followed by incubation with HRP-coupled anti-mouse (Dako) or anti-rabbit (Dako) secondary antibodies. In some experiments, patient sera were used as primary antibody sources, followed by incubation with HRP-coupled anti-human IgG (Dako). Individual bands were visualized with ECL (GE Healthcare). Quantification was done using Quantity One 1-D Analysis software (Bio-Rad).

Histopathological examination. H\&E- and Gomori trichromestained muscle biopsy sections from 20 anti-FHL1 ${ }^{+}$patients and 13 sex-, age-, and diagnosis-matched (PM, DM, and IBM) anti-FHL1patients were available in the muscle pathology repository at the Neuromuscular unit of Karolinska University Hospital and were assessed by an experienced muscle pathologist. Scoring was done blindly with regard to the status of anti-FHL1 autoantibody positivity. Characteristics examined included muscle fiber atrophy, fibrosis, necrosis/degeneration, regeneration, presence of inflammatory infiltrates, internal nuclei, variation of fiber size, and rimmed vacuoles. Overall histopathological severity was scored using a VAS system $(0-10 \mathrm{~cm})(31)$.

Immunofluorescense and confocal microscopy. Muscle biopsies were frozen in isopenthane prechilled in liquid nitrogen, sectioned into $7-\mu \mathrm{m}$ sections, formalin fixed, and stored at $-70^{\circ} \mathrm{C}$ until processing. Formalin-fixed muscle tissue sections were permeabilized with $0.1 \%$ saponin and stained with monoclonal mouse anti-human FHL1 antibody (dilution 1:200; catalog ab76912; Abcam), followed by Alexa Fluor 594-conjugated anti-mouse antibody (Invitrogen). Moreover, unfixed healthy human muscle tissue was stained with sera from anti-FHL1 ${ }^{+}$and anti-FHL1 ${ }^{-}$patients as well as with sera from HCs (dilution 1:50), followed by incubation with DyLight 594-conjugated donkey-anti human IgG antibody (Pierce, Thermo Scientific). In some experiments, the positive staining using either commercial anti-FHL1 or anti-FHL1 ${ }^{+}$human sera was blocked by preincubation with recombinant FHL1-MaBP protein overnight at $4^{\circ} \mathrm{C}$. In addition, sections were incubated with rabbit polyclonal anti-laminin, followed by Alexa Fluor 488-conjugated anti-rabbit antibody (Invitrogen). Nuclei were counterstained with DAPI (Roche), and sections were mounted in Fluoromount-G (SouthernBiotech). Images were acquired using a Leica TCS SP5, and acquisition was performed using a $\times 40$ oil-immersion objective. A $z$-dimension series was taken every $0.2 \mu \mathrm{m}$. Images were analyzed with Image J software (NIH).

$m R N A$ expression analysis and $q P C R$. For studies with human material, RNA was obtained from muscle biopsies from healthy individuals and myositis patients positive or negative for anti-FHL1 autoantibodies. The RNeasy Fibrous Tissue Mini Kit (QIAGEN) was used for RNA extraction. cDNA synthesis was performed with an iScript cDNA synthesis kit (Bio-Rad) following the manufacturer's protocol. Predesigned and custom TaqMan assays from Life Technologies were used to interrogate the transcript expression levels of 3 well-established splice isoforms of FHL1 (reviewed in ref. 20), as represented by 
their NCBI Reference Sequence (RefSeq) accession numbers: FHL1A, NM_001449.4 (full-length); FHL1B, NM_001159702.2; and FHL1C, NM_001159703.1. Detection of the FHL1B transcript was performed using the predesign real-time gene expression assay Hs00938359 g1 (Applied Biosystems, Life Technologies), with a probe spanning exons 7-8; the context sequence reporter was $5^{\prime}$-FAM ${ }^{*}$-CTCCTCGAGGCCCGGGTTTGGTAAA-NFQ ${ }^{* *}-3^{\prime}$. Two custom TaqMan assays were designed to evaluate the expression of FHL1A and FHL1B transcripts. For the FHL1A transcript, the context sequence spanning exons 6-8 was 5'-FAM ${ }^{*}$-ACCCCATCACTGGGTTTGGTA-NFQ ${ }^{* *}-3^{\prime}$, and for the FHL1C transcript, the probe spanning exons 5-8 was 5'-FAM ${ }^{*}$-AGTGCAACAAGGGTTTGG-NFQ ${ }^{* *}-3^{\prime}$. The experiment was performed at least in triplicate. Three nontemplate controls were included in each experiment. Expression measurements were made using the $\Delta \Delta$ relative quantification method $(\Delta \Delta \mathrm{Ct})$ with QuantStudio6 and the 7 Flex Real-Time PCR System and ExpressionSuite Software, version 1.0.4 (all from Thermo Fisher Scientific). The ZNF592 gene was used as an endogenous reference control (predesign assay Hs00206029_m1), the $\Delta \mathrm{Ct}$ of which was subtracted from each individual measurement. The mean $\Delta \mathrm{Ct}$ values for controls were used as a calibrator.

For the animal experiment, cDNA was prepared from hind-limb muscle tissue using the Applied Biosystems High-Capacity cDNA Reverse Transcription Kit (Life Technologies; catalog 43-688-14). Total RNA (800 ng) was treated with DNAse and reverse transcribed. cDNA (40 ng) was used per $10 \mu \mathrm{l}$ qPCR reaction. The following assays were selected from the Life Technologies TaqMan repository: Cd4 (Mm00442754_m1 ); Cd8a (Mm01188922_m1); Gzmb (Mm00442834_m1); and Prf1 (Mm00812512_m1).

Cleavage assays of muscle lysates. Cultured skeletal muscle cells were lysed in 10 mM HEPES, 2 mM EDTA, 1\% NP-40 including leupeptin, antipain, pepstatin A, and phenylmethylsulfonyl fluoride (PMSF), and human recombinant granzyme B (all from Enzo Life Sciences) was used in different concentrations for 1.5 hours at $37^{\circ} \mathrm{C}$. As indicated in Figure 5B, 5 mM DTT (Sigma-Aldrich), $500 \mathrm{nM} \mathrm{z}$-Vad-FMK, or $500 \mathrm{nM}$ z-DEVD-FMK (both from BD Pharmingen) was added. In some experiments, 100 ng recombinant protein was used as a substrate. The reaction was loaded onto an SDS-PAGE gel and blotted onto a nitrocellulose membrane. Detection was done with a mouse monoclonal anti-FHL1 antibody (ab76912; Abcam, binding to the N-terminus) or a goat polyclonal anti-FHL1 (ab23937; Abcam, binding to the C-terminus). The granzyme B cleavage site in FHL1 was confirmed by cloning FHL1 in pTNT plasmid (Promega) and mutating aspartic acid (D) at amino acid position 50 to alanine (A) using site-directed mutagenesis (Agilent Technologies). Primers used for mutagenesis PCR were a149c antisense (5'-CACCTCCTTGGAGGCCGCACCGATGGG-3') and a149c sense (5'-CCCATCGGTGCGGCCTCCAAGGAGGTG-3'). WT and mutated protein were expressed by in vitro transcription/translation (IVTT) (Promega), cleaved with granzyme B, and analyzed by immunoblotting.

HLA genotyping. HLA genotyping was performed using sequencespecific primer PCR (SSP-PCR) (DR low-resolution kit; Olerup SSP), and the PCR products were loaded onto $2 \%$ agarose gels for electrophoresis. An interpretation table was used to determine the specific genotype according to the manufacturer's recommendations (62).

Mass-spectrometric analysis. Bands identified by Western blotting as containing FHL1 were excised from a Coomassie-stained gel (SDSPAGE). The bands were reduced, alkylated, and digested by trypsin using standard protocols (63). After extraction and desalting using ziptips (Merck Millipore), the peptides were analyzed using on-line nanoflow liquid chromatography, with a C18 reverse-phase column coupled to an LTQ Orbitrap Velos ETD mass spectrometer (Thermo Fisher Scientific). Mass lists were extracted from the raw data using Raw2MGF (64) and searched against the human complete proteome database (2013/14) using the Mascot search engine, v2.4.1 (Matrix Science Ltd.). The following parameters were used for the database search: tryptic digestion (maximum of 2 miscleavages); carbamethylation $(\mathrm{C})$ as a fixed modification; oxidation $(\mathrm{M})$, pyroglutamate $(\mathrm{Q})$ as the variable; $10 \mathrm{ppm}$ as the precursor tolerance; and $0.25 \mathrm{Da}$ as the fragment tolerance. MudPIT scoring was used to evaluate the results.

Immunization of HT mice. The mouse model of myositis used, including genotyping of the mice, was described previously (35). Briefly, the model uses a tet-off-driven expression of the $\mathrm{H}-2 \mathrm{~Kb}$ MHC class I molecule (H gene). Doxycycline was withdrawn from the water at 5 weeks of age to induce expression of MHC class I. To study the effect of FHL1 on development of myositis, female HT $(n=8)$ and age-matched $\mathrm{H}$ mice $(n=8)$ were immunized s.c. with $250 \mu \mathrm{g}$ recombinant FHL1-MaBP fusion protein in CFA. For additional controls, age-matched female HT $(n=8)$ and H mice $(n=8)$ were immunized with recombinant MaBP in CFA. Two weeks after immunization, mice received a booster consisting of $125 \mu \mathrm{g}$ FHL1 or MaBP in incomplete Freund's adjuvant (IFA). Blood was collected routinely at 2, 6, and 10 weeks after immunization and at the time of euthanization. Nine weeks after immunization, BW was determined and grip strength for both forelimbs and hind limbs was assessed using a grip strength meter as described elsewhere (65). Sixteen weeks after immunization, mice were euthanized. Survival is shown using a Kaplan Meier survival plot (Figure 6E). To analyze muscle injury, IgM staining was performed as described previously (66).

Statistics. The significance of differences in the ELISAs, clinical outcome scores, histopathology scores, as well as grip strength and weight measurements in the animal studies were calculated by Mann-Whitney $U$ tests. The significance of differences between anti$\mathrm{FHL}^{+}$and anti-FHL ${ }^{-}$patients was calculated by the Wilcoxon ranksum test for continuous variables and by Pearson's $\chi^{2}$ or Fischer's exact test for categorical variables. Logistic regression analysis was used for assessment of the probability of having anti-FHL1 autoantibodies for different categories of patients. Stata (StatCorp) or GraphPad Prism 6.0 (GraphPad Software) were used for data management and statistical analyses. $P$ values of 0.05 or less were considered statistically significant.

Study approval. Collection of serum and biopsies from IIM patients as well as serum from patients with other autoimmune diseases was approved by the local ethics committee of Karolinska Institutet. Collection of serum from IIM patients from the Czech Republic was approved by the Institute of Rheumatology in Prague. All patients provided informed consent before their participation in this study.

All handling and experimentation with mice was approved by the IACUC of the Children's National Medical Center in Washington, DC.

\section{Author contributions}

IA designed the study, conducted and analyzed the experiments, and wrote the manuscript. CW contributed to study design and, with $\AA \mathrm{H}$, conducted muscle cDNA library experiments and initial ELISAs. AT performed statistical analysis of clinical data and edited the manuscript. KN, KT, AP, and WC designed and conducted the 
animal study and edited the manuscript. FA designed the granzyme B experiments and edited the manuscript. LMDG designed and performed the qPCRs for the different FHL1 isoforms. MB was the statistician and IN the pathologist for the study. $\mathrm{KC}$ and JH provided scientific input and edited the manuscript. KJ and AW contributed to clinical data abstraction for the Swedish cohort. AJY and RAZ conducted the mass spectrometric experiments. OD provided samples and clinical data from patients with NMD. O Krystufkova and JV provided patient samples and clinical data for the Czech cohort and edited the manuscript. NL conducted in vitro experiments. MWH contributed to study design and provided scientific input. LP contributed to study design and analyzed HLA associations. O Kämpe contributed to study design and provided scientific input. IEL designed the study and edited the manuscript.

\section{Acknowledgments}

We thank Elisabet Svenungsson and Annika Nordin for providing us with patient sera, Eva Jemseby for careful handling of the serum bank, Eva Lindroos and Barbro Larsson for their excellent techni- cal assistance, nurse Christina Ottosson for her excellent patient care, Maryam Dastmalchi for collecting data for the myositis registries, Megan K. Herbert for providing data on $\mathrm{cN}-1 \mathrm{~A}$ reactivity in our patient cohort, and Lars Klareskog and Vivianne Malmström for their critical reading of the manuscript. This work was supported by grants from the Swedish Research Council, the Torsten and Ragnar Söderberg Foundations, the Karolinska Institutet, the Börje Dahlin Foundation, the Swedish Rheumatism Association, the King Gustaf V 80-year Foundation, the regional agreement between Stockholm County Council and Karolinska Institutet on medical training and clinical research (ALF), and the Swedish Strategic Foundation (PRIMI).

Address correspondence to: Inka Albrecht or Ingrid E. Lundberg, Karolinska Institutet, Department of Medicine, Rheumatology Unit, Karolinska Hospital Solna, SE 17176 Stockholm, Sweden. Phone: 46.85.177.0310; E-mail: inka.albrecht@ki.se (I. Albrecht). Phone: 46.85.177.6087; E-mail: ingrid.lundberg@ ki.se (I.E. Lundberg).
1. Dalakas MC, Sivakumar K. The immunopathologic and inflammatory differences between dermatomyositis, polymyositis and sporadic inclusion body myositis. Curr Opin Neurol. 1996;9(3):235-239.

2. Ernste FC, Reed AM. Idiopathic inflammatory myopathies: current trends in pathogenesis, clinical features, and up-to-date treatment recommendations. Mayo Clin Proc. 2013;88(1):83-105.

3. Emslie-Smith AM, Engel AG. Necrotizing myopathy with pipestem capillaries, microvascular deposition of the complement membrane attack complex (MAC), and minimal cellular infiltration. Neurology. 1991;41(6):936-939.

4. Christopher-Stine L, Casciola-Rosen LA, Hong G, Chung T, Corse AM, Mammen AL. A novel autoantibody recognizing 200-kd and 100-kd proteins is associated with an immune-mediated necrotizing myopathy. Arthritis Rheum. 2010;62(9):2757-2766.

5. Salajegheh M, Lam T, Greenberg SA. Autoantibodies against a $43 \mathrm{KDa}$ muscle protein in inclusion body myositis. PLoS One. 2011;6(5):e20266.

6. Larman HB, et al. Cytosolic 5'-nucleotidase 1A autoimmunity in sporadic inclusion body myositis. Ann Neurol. 2013;73(3):408-418.

7. Pluk H, et al. Autoantibodies to cytosolic 5 '-nucleotidase $1 \mathrm{~A}$ in inclusion body myositis. Ann Neurol. 2013;73(3):397-407.

8. Ray A, et al. Autoantibodies produced at the site of tissue damage provide evidence of humoral autoimmunity in inclusion body myositis. PLOS One. 2012;7(10):e46709.

9. Gunawardena H, Betteridge ZE, McHugh NJ. Myositis-specific autoantibodies: their clinical and pathogenic significance in disease expression. Rheumatology. 2009;48(6):607-612.

10. Targoff IN. Myositis specific autoantibodies. Curr Rheumatol Rep. 2006;8(3):196-203.

11. Betteridge ZE, Gunawardena H, McHugh NJ. Novel autoantibodies and clinical phenotypes in adult and juvenile myositis. Arthritis Res Ther. 2011;13(2):209.
12. Cowling BS, et al. Identification of FHL1 as a regulator of skeletal muscle mass: implications for human myopathy. JCell Biol. 2008;183(6):1033-1048.

13. McGrath MJ, Mitchell CA, Coghill ID, Robinson PA, Brown S. Skeletal muscle LIM protein 1 (SLIM1/FHL1) induces $\alpha 5 \beta 1$-integrin-dependent myocyte elongation. Am J Physiol Cell Physiol. 2003;285(6):C1513-C1526.

14. Wilding BR, McGrath MJ, Bonne G, Mitchell CA. FHL1 mutants that cause clinically distinct human myopathies form protein aggregates and impair myoblast differentiation. J Cell Sci. 2014;127(pt 10):2269-2281.

15. McGrath MJ, et al. Four and a half LIM protein 1 binds myosin-binding protein $\mathrm{C}$ and regulates myosin filament formation and sarcomere assembly. J Biol Chem. 2006;281(11):7666-7683.

16. Ding L, et al. Human four-and-a-half LIM family members suppress tumor cell growth through a TGF- $\beta$-like signaling pathway. JClin Invest. 2009;119(2):349-361.

17. Lin J, et al. Four and a half LIM domains 1 (FHL1) and receptor interacting protein of $140 \mathrm{kDa}$ (RIP140) interact and cooperate in estrogen signaling. Int J Biochem Cell Biol. 2009;41(7):1613-1618.

18. Qin H, Wang J, Liang Y, Taniguchi Y, Tanigaki K, Han H. RING1 inhibits transactivation of RBP-J by Notch through interaction with LIM protein KyoT2. Nucleic Acids Res. 2004;32(4):1492-1501.

19. Sheikh F, et al. An FHL1-containing complex within the cardiomyocyte sarcomere mediates hypertrophic biomechanical stress responses in mice. JClin Invest. 2008;118(12):3870-3880.

20. Shathasivam T, Kislinger T, Gramolini AO. Genes, proteins and complexes: the multifaceted nature of FHL family proteins in diverse tissues. JCell Mol Med. 2010;14(12):2702-2720.

21. Schessl J, et al. Familial reducing body myopathy with cytoplasmic bodies and rigid spine revisited identification of a second LIM domain mutation in FHL1. Neuropediatrics. 2010;41(1):43-46.

22. Schessl J, et al. Clinical, histological and genetic characterization of reducing body myopathy caused by mutations in FHL1. Brain. 2009;132(pt 2):452-464.

23. Schessl J, et al. Proteomic identification of FHL1 as the protein mutated in human reducing body myopathy. JClin Invest. 2008;118(3):904-912.

24. Shalaby S, Hayashi YK, Nonaka I, Noguchi S, Nishino I. Novel FHL1 mutations in fatal and benign reducing body myopathy. Neurology. 2009;72(4):375-376.

25. Schoser B, et al. Consequences of mutations within the C terminus of the FHL1 gene. Neurology. 2009;73(7):543-551.

26. Windpassinger C, et al. An X-linked myopathy with postural muscle atrophy and generalized hypertrophy, termed XMPMA, is caused by mutations in FHL1. Am J Hum Genet. 2008;82(1):88-99.

27. Chen DH, et al. A novel mutation in FHL1 in a family with X-linked scapuloperoneal myopathy: phenotypic spectrum and structural study of FHL1 mutations. J Neurol Sci. 2010;296(1-2):22-29.

28. Gueneau L, et al. Mutations of the FHL1 gene cause Emery-Dreifuss muscular dystrophy. Am J Hum Genet. 2009;85(3):338-353.

29. Cowling BS, Cottle DL, Wilding BR, D'Arcy CE, Mitchell CA, McGrath MJ. Four and a half LIM protein 1 gene mutations cause four distinct human myopathies: a comprehensive review of the clinical, histological and pathological features. Neuromuscul Disord. 2011;21(4):237-251.

30. Quinzii CM, et al. X-linked dominant scapuloperoneal myopathy is due to a mutation in the gene encoding four-and-a-half-LIM protein 1. Am J Hum Genet. 2008;82(1):208-213.

31. Wedderburn LR, et al. International consensus on a proposed score system for muscle biopsy evaluation in patients with juvenile dermatomyositis: a tool for potential use in clinical trials. Arthritis Rheum. 2007;57(7):1192-1201.

32. Darrah E, Rosen A. Granzyme B cleavage of autoantigens in autoimmunity. Cell Death Differ. 2010;17(4):624-632.

33. Thornberry NA, et al. A combinatorial approach 
defines specificities of members of the caspase family and granzyme B. J Biol Chem. 1997;272(29):17907-17911.

34. Mahrus S, Craik CS. Selective chemical functional probes of granzymes $A$ and $B$ reveal granzyme $B$ is a major effector of natural killer cell-mediated lysis of target cells. Chem Biol. 2005;12(5):567-577.

35. Nagaraju K, et al. Conditional up-regulation of MHC class I in skeletal muscle leads to selfsustaining autoimmune myositis and myositisspecific autoantibodies. Proc Natl Acad Sci US A. 2000;97(16):9209-9214.

36. Zhang D, Beresford PJ, Greenberg AH, Lieberman J. Granzymes A and B directly cleave lamins and disrupt the nuclear lamina during granulemediated cytolysis. Proc Natl Acad Sci U S A. 2001;98(10):5746-5751.

37. Zhu P, Martinvalet D, Chowdhury D, Zhang D, Schlesinger A, Lieberman J. The cytotoxic T lymphocyte protease granzyme A cleaves and inactivates poly(adenosine $5^{\prime}$-diphosphate-ribose) polymerase-1. Blood. 2009;114(6):1205-1216.

38. Casciola-Rosen L, Andrade F, Ulanet D, Wong $\mathrm{WB}$, Rosen A. Cleavage by granzyme B is strongly predictive of autoantigen status: implications for initiation of autoimmunity. J Exp Med. 1999;190(6):815-826.

39. Nagaraju K, Cox A, Casciola-Rosen L, Rosen A. Novel fragments of the Sjogren's syndrome autoantigens alpha-fodrin and type 3 muscarinic acetylcholine receptor generated during cytotoxic lymphocyte granule-induced cell death. Arthritis Rheum. 2001;44(10):2376-2386.

40. Andrade F, Roy S, Nicholson D, Thornberry N, Rosen A, Casciola-Rosen L. Granzyme $B$ directly and efficiently cleaves several downstream caspase substrates: implications for CTL-induced apoptosis. Immunity. 1998;8(4):451-460.

41. Huang $\mathrm{M}$, et al. La autoantigen translocates to cytoplasm after cleavage during granzyme B-mediated cytotoxicity. Life Sci. 2007;81(19-20):1461-1466.

42. Chen CN, Ferrington DA, Thompson LV. Carbonic anhydrase III and four-and-a-half LIM protein 1 are preferentially oxidized with muscle unloading. J Appl Physiol (1985).
2008;105(5):1554-1561.

43. Gono T, et al. Brief report: Association of HLA-DRB $1{ }^{*} 0101 /{ }^{*} 0405$ with susceptibility to anti-melanoma differentiation-associated gene 5 antibody-positive dermatomyositis in the Japanese population. Arthritis Rheum. 2012;64(11):3736-3740.

44. Chinoy $\mathrm{H}$, et al. Interaction of HLA-DRB $1^{*} 03$ and smoking for the development of anti-Jo- 1 antibodies in adult idiopathic inflammatory myopathies: a European-wide case study. Ann Rheum Dis. 2012;71(6):961-965.

45. Mammen AL, et al. Increased frequency of DRB1*11:01 in anti-hydroxymethylglutarylcoenzyme A reductase-associated autoimmune myopathy. Arthritis Care Res (Hoboken). 2012;64(8):1233-1237.

46. Miller FW, et al. Genome-wide association study of dermatomyositis reveals genetic overlap with other autoimmune disorders. Arthritis Rheum. 2013;65(12):3239-3247.

47. Dalakas MC. Polymyositis, dermatomyositis and inclusion-body myositis. $N$ Engl J Med. 1991;325(21):1487-1498.

48. Dalakas MC. Mechanisms of disease: signaling pathways and immunobiology of inflammatory myopathies. Nat Clin Pract Rheumatol. 2006;2(4):219-227.

49. Dalakas MC. Pathogenesis and therapies of immune-mediated myopathies. Autoimmun Rev. 2012;11(3):203-206.

50. Dalakas MC, Hohlfeld R. Polymyositis and dermatomyositis. Lancet. 2003;362(9388):971-982.

51. Carpenter JR, Bunch TW, Engel AG, O'Brien PC. Survival in polymyositis: corticosteroids and risk factors. J Rheumatol. 1977;4(2):207-214.

52. Horowitz M, McNeil JD, Maddern GJ, Collins PJ, Shearman DJ. Abnormalities of gastric and esophageal emptying in polymyositis and dermatomyositis. Gastroenterology. 1986;90(2):434-439.

53. Bohan A, Peter JB. Polymyositis and dermatomyositis (second of two parts). N Engl JMed. 1975;292(8):403-407.

54. Bohan A, Peter JB. Polymyositis and dermatomyositis (first of two parts). N Engl J Med. 1975;292(7):344-347.

55. Griggs RC, et al. Inclusion body myositis and myopathies. Ann Neurol. 1995;38(5):705-713.

56. Isenberg DA, et al. International consensus outcome measures for patients with idiopathic inflammatory myopathies. Rheumatology (Oxford). 2004;43(1):49-54.

57. Bei R, Masuelli L, Palumbo C, Modesti M, Modesti A. A common repertoire of autoantibodies is shared by cancer and autoimmune disease patients: Inflammation in their induction and impact on tumor growth. Cancer Lett. 2009;281(1):8-23.

58. Lleo A, Invernizzi P, Gao B, Podda M, Gershwin ME. Definition of human autoimmunity - autoantibodies versus autoimmune disease. Autoimmun Rev. 2010;9(5):A259-A266.

59. Schmidt J, Dalakas MC. Inclusion body myositis: from immunopathology and degenerative mechanisms to treatment perspectives. Exp Rev Clin Immunol. 2013;9(11):1125-1133.

60. Dimachkie MM, Barohn RJ. Inclusion body myositis. Neurol Clin. 2014;32(3):629-646.

61. Salomonsson S, Dorner T, Theander E, Bremme $\mathrm{K}$, Larsson P, Wahren-Herlenius M. A serologic marker for fetal risk of congenital heart block. Arthritis Rheum. 2002;46(5):1233-1241.

62. Lundstrom E, et al. Opposing effects of HLA$\mathrm{DRB1}^{*} 13$ alleles on the risk of developing anti-citrullinated protein antibody-positive and anti-citrullinated protein antibody-negative rheumatoid arthritis. Arthritis Rheum. 2009;60(4):924-930.

63. Shevchenko A, Wilm M, Vorm O, Mann M. Mass spectrometric sequencing of proteins silver-stained polyacrylamide gels. Anal Chem. 1996;68(5):850-858.

64. Lyutvinskiy Y, Yang H, Rutishauser D, Zubarev RA. In silico instrumental response correction improves precision of label-free proteomics and accuracy of proteomics-based predictive models. Mol Cell Proteomics. 2013;12(8):2324-2331.

65. Coley W, Rayavarapu S, van der Meulen JH, Duba AS, Nagaraju K. Daily supplementation of D-ribose shows no therapeutic benefits in the MHC-I transgenic mouse model of inflammatory myositis. PLoS One. 2013;8(6):e65970.

66. Jahnke VE, et al. Metabolic remodeling agents show beneficial effects in the dystrophin-deficient mdx mouse model. Skelet Muscle. 2012;2(1):16. 